\title{
Prótesis Robóticas y Pieles Dinámicas: Programación Visual Y Código Abierto Como Integración Contextual De Sistemas Industriales Cerrados
}

\author{
SEBASTIÁN GIRALDI \\ Universidad Nacional del Litoral \\ RODRIGO GARCÍA ALVARADO \\ Universidad del BIO-BIO
}

\author{
MAURO CHIARELLA \\ CONICET-Universidad Nacional del Litoral \\ BRAULIO GATICA \\ Universidad del BIO-BIO
}

To the aim of providing comprehensive architectural response targeted to programming changing occupation of buildings, based on the periodical behavior but also free control of users to combine the analysis of the existing building with a device interface mode applied to dynamic industrialized lattices. This experience of contextual adaptation evidence the maker culture, as a strategy of localization of the global.

\section{PIELES DINÁMICAS}

Manuel Gauza ha definido la piel arquitectónica como una capa exterior mediadora entre el edificio y su entorno. La sustitución del concepto de fachada por el de piel sugiere pensarla como una membrana activa, informada, comunicada y comunicativa antes que un alzado neutro derivado de una simple representación abstracta ${ }^{1}$. La complejidad del nuevo concepto radica en admitir la superposición de elementos funcionales e instalaciones con la capacidad de captar, administrar y transmitir energía.

La arquitectura se ha caracterizado por generar espacialidades estables, sólidas, corpóreas. En las últimas décadas hay un marcado interés por abordar la pérdida de la materialidad y la incorporación de situaciones espaciales dinámicas, variables y flexibles. Al incorporar tecnologías innovadoras en la ideación, desarrollo y manufactura, es posible reinventar un clásico elemento constituyente de lo arquitectónico como es el muro. Es así como tradicionales características y objetivos referidos al muro están siendo redefinidos: lo estático se vuelve fluído, lo opaco se vuelve transparente, la barrera se vuelve filtro y los límites se vuelven neutrales. La tecnología ha permitido al muro convertirse en un dinámico y sensible componente del ambiente construido. Osman Ataman ${ }^{2}$ describe los avances alcanzados hasta hoy en la evolución de los sistemas de muros arquitectónicos que incorporan sensores en los edificios, combinando materiales, sistemas inteligentes, ingeniería y arte se pueden crear componentes que no solo soportan y definen un espacio sino que lo trasciende enriqueciéndolo. En la arquitectura contemporánea podemos clasificar, según sus cualidades y prestaciones: muros de geometrías curvas y complejas, muros dinámicos, muros responsivos, muros interactivos, muros inteligentes.

Entre los diferentes términos utilizados para describir la zona entre el exterior arquitectónico y su interior, existen ciertas diferencias entre lo que se conoce como: envolvente, pared, muro, piel y fachada. Envolvente es un término genérico que describe el cierre total de un edificio. Pared se ha utilizado tradicionalmente para describir una construcción de soporte vertical. Muro cortina es un término que aparece a principios del siglo XX para distinguir una construcción que no soporta carga. Piel es un término acuñado inicialmente para continuar con la distinción entre el revestimiento y la estructura de una pared, pero ha sido más recientemente asociada como un sistema ambiental inteligente. La fachada se asocia como parte de una composición urbana.

La evolución del Muro a la Piel Dinámica es aún una materia pendiente en la enseñanza de la arquitectura en Latinoamérica. Un muro podría ser más que una barrera o estructura, puede ser un sistema integrado y completo. Dentro de las prácticas y enseñanza arquitectónica observamos la ausencia de ejercicios proyectuales que incorporen estrategias didácticas para imaginar geometrías dinámicas, flexibles, como estructura posibilitante de las pieles sensibles propuestas. Nuestra formación y práctica disciplinar aún concibe geométricamente desde la rigurosidad y estabilidad modular que nos proponen las geomerías en un estado pasivo, determinante de una única situación espacial y morfológica. Nos preguntamos: ¿Cómo se pensaría la geometría de un muro de modo dinámico si convencionalmente se lo ha considerado como algo estable? ¿qué instrumentos proyectuales de generación, adaptación, sustitución o integración con sistemas existentes poseemos para abordarlas?

A partir de esta problemática, se han desarrollado prototipos a escala mediante microcontroladores y patrones plegados (Chiarella, Raffin, 

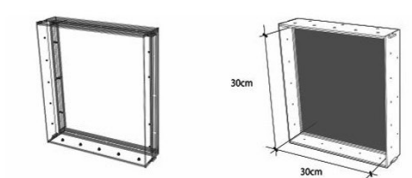
$30 \mathrm{~cm}-1$

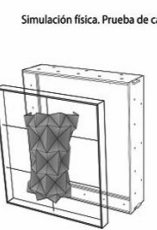
Opción 1 - Compresion en los
extremos staterales
ysuperior e einterior

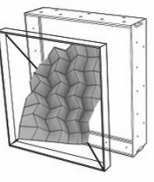

Opción 2 -Tensionen los
cuatros extremsos alternand

cuatros extremss, alternando
la fuerza de influencia
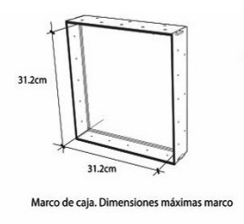
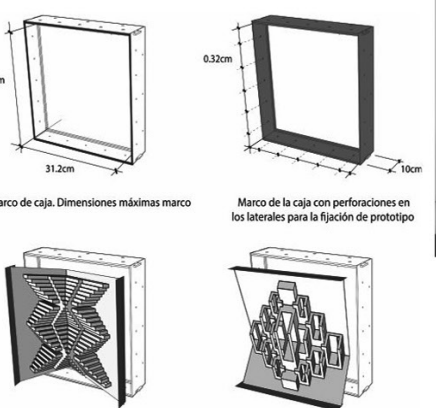

Opción 3 - Rigidezen les
latereses izavierdoy derecho
Retroimiento de los lados

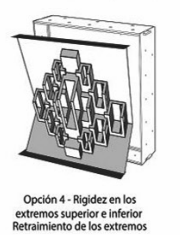

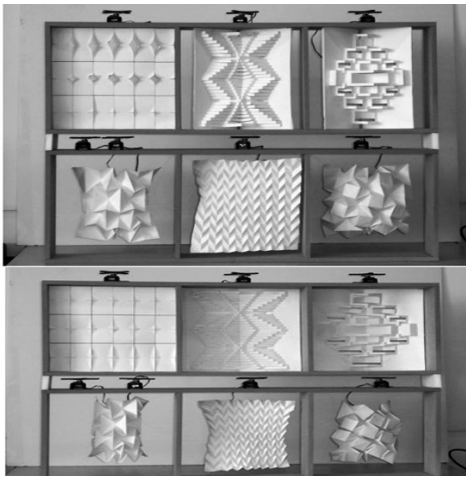

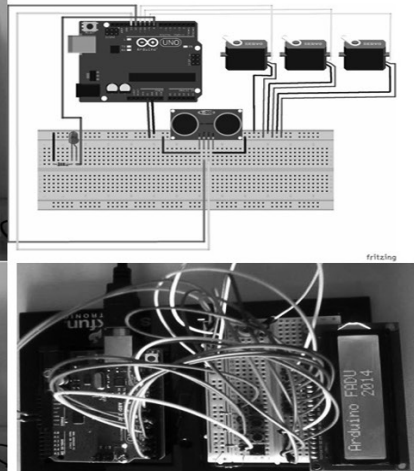

Figura 1: Prototipos a escala de Pieles Dinámicas (Arduino FADU-UNL)

Góngora, Martini; 2014) ${ }^{3}$ integrados a dispositivos portátiles de adquisición de datos para eficiencia energética (Chiarella, Góngora; 2015). En esta oportunidad nos ha interesado trabajar sobre una integración de bajo costo de sistemas comerciales industrializados de código cerrado. Las variables contextuales que constituyen la nueva problemática derivan del resultado de una evaluación post-ocupación edilicia (POE).

\section{REACTIVO O DINÁMICO}

El diseño reactivo está vinculado a la activación por estímulos. Los sistemas reactivos son elementos de nuestro entorno que tienen (en diversos niveles de complejidad) una gama de contenidos predefinidos, como comportamientos que se pueden activar para producir un cambio. La mayor parte de diseño de interacción hoy es una mejora estética de los modelos de diseño reactivo enraizada en los primeros métodos de instrucción en computación; un modelo rígido, lineal y algo restrictivo.

Ruairi Glynn ${ }^{4}$ sugiere que es más útil y productivo entender la interactividad como un modelo de dialogo o conversación, tal como naturalmente, ocurre entre los seres humanos o en el reino animal en general. La interactividad sería entonces como una actividad de conversación entre los usuarios y el objeto que se ha diseñado, en cambio un modelo reactivo funciona haciendo caso de rígidas restricciones previamente diseñadas e incorporadas en el objeto donde el comportamiento es orientado por los comandos conteniendo ciertas restricciones controladas preestablecidas. Michael Fox ${ }^{5}$ también argumenta a favor de la arquitectura dinámica en las cuales los sistemas tienen capacidad de aprendizaje y funcionan de acuerdo a un modelo conversacional. Fox (Kinetic Design Group, MIT) ha publicado los principios de los sistemas de comportamiento dinámicos identificando seis tipos ordenados por nivel de complejidad.

El concepto de responsivo se utiliza en un sentido general para describir una interacción entre un sistema dinámico y el entorno, incluye la interacción humana para generar una respuesta directa, además de reacción natural a una fuerza del medio ambiente aleatoria. De esta manera vemos que la interacción no se limita a los comportamientos rígidos de los sistemas reactivos. Como una extensión de esto, si a los artefactos diseñados se les da la capacidad de improvisar, negociar y aprender de ellos mismos, tienen el potencial, a través de la interacción con otros participantes de desarrollar sus propios comportamientos personales

más allá de las nociones preconcebidas de su diseñador personal y así se convierten en objetos responsivos. Tal proceso evolutivo logra dotar a las pieles arquitectónicas de comportamientos y expresiones aumentando sus capacidades expresivas y de adaptación y eficiencia en relación a factores ambientales externos. El sistema elegido para nuestra investigación es un sistema reactivo automatizado.

\section{PRÓTESIS ROBÓTICAS}

Una prótesis robótica es un elemento artificial dotado de cierta autonomía e inteligencia capaz de realizar una función de una parte faltante del cuerpo humano. Dicha autonomía e inteligencia se logra al integrar sensores, procesadores, actuadores y complejos algoritmos de control.

\section{INTEGRACIÓN}

Integración contextual de Domótica de Código Cerrado. Con el objetivo de dar una respuesta integral que ponga como centro de la programación a las conductas vivenciales y cambiantes de los ocupantes de un edificio, se aplica un sistema basado en encuestas a usuarios, mandantes y fabricantes, que combina el análisis del edificio existente con un dispositivo a modo de interface de celosías dinámicas industrializadas. (arduino, grasshopper, firefly).

\section{ENCUESTA DE PERCEPCIÓN USUARIOS CITEC}

Muchos edificios no se llevan a cabo como estaban previsto o sufren modificaciones sobre la marcha, teniendo un impacto en los costes de funcionamiento, el usuario, el rendimiento, la salud, la seguridad y el confort. La evaluación post-ocupación (POE) es el proceso de obtención de información sobre el rendimiento de un edificio en uso ${ }^{6}$. El valor de POE está siendo cada vez más reconocido, y se está convirtiendo en seguimientos obligatorios en muchos proyectos públicos. POE es valiosa en todos los sectores de la construcción, especialmente salud, educación, oficinas, comercial y vivienda, donde los malos resultados tendrán un impacto en los costes de funcionamiento, el bienestar del usuario y en la eficiencia energética edilicia. La evaluación post-ocupación realizada a los usuarios del edificio CITEC (Universidad del Bio-Bio, Concepción-Chile) en relación a las celosías automatizadas de control solar ha tenido los siguientes objetivos:

a) Destacar los problemas iniciales inmediatos que pueden ser abordados y solucionados; 
b) Identificar las lagunas en la comunicación y la comprensión del impacto en las formas de operar de los sistemas propuestos en edificio

c) La consulta de los ocupantes en relación a la comodidad y el control sobre las condiciones ambientales que inciden en la eficiencia energética edilicia

d) Proporcionar lecciones que se pueden utilizar para mejorar el diseño y la contratación en proyectos futuros

\section{PRÓTESIS ROBÓTICAS DE CÓDIGO ABIERTO:}

Este desarrollo parte como una aproximación a establecer herramientas de análisis y una propuesta de solución para factores de Post-Ocupación (en inglés Post-Occupancy Evaluations - POE) o

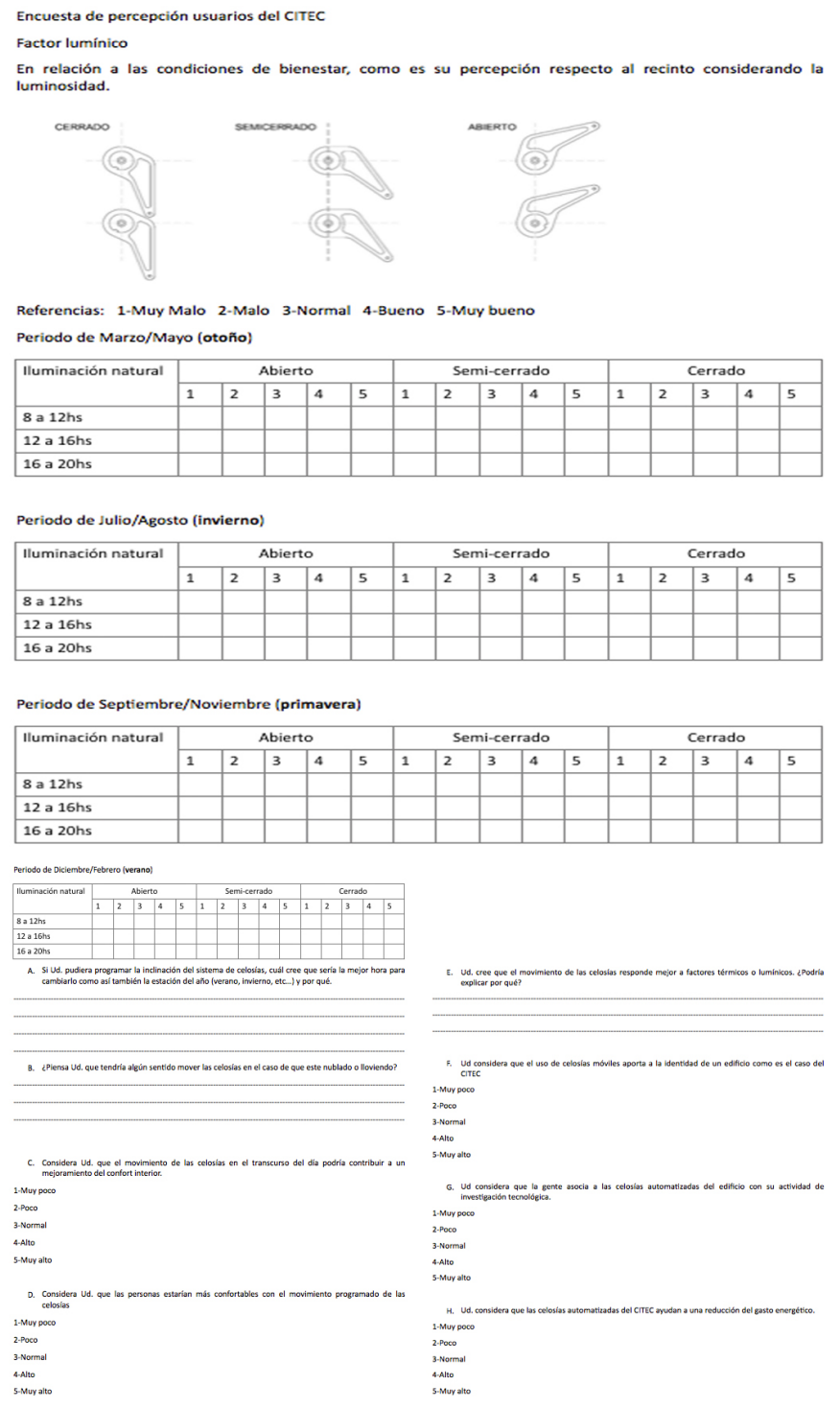

Figure 2: Encuesta de percepción usuarios CITEC (Giraldi, Sebastían) sea, la manera de determinar cómo se está usando un edificio y que problemas se encuentran. La solución al problema encontrado fue el diseño y construcción de un dispositivo híbrido (prótesis robótica) que busca establecer un dialogo entre sistemas de programación open source y sistemas industriales de hardware y software cerrado.

La programación se hizo en el entorno de Grasshopper (plug-in de Rhino) y utilizando Firefly que es un conjunto de herramientas que opera sobre Grasshopper. Dichas herramientas permiten conectar en tiempo real la programación digital de grasshopper con el hardware, que en este caso es un microcontrolador Arduino. Es así como la placa Arduino viene a funcionar como interface entre la programación digital y el modelo físico que son los dedos robóticos, los cuales van a operar sobre el control remoto que permite la apertura o cierre de las celosías Hunter Douglas. En cuanto a la descripción de la programación se puede describir en los siguientes pasos;

1) Establece la comunicación entre el computador y el encendido de la placa Arduino

2) Lee la hora del computador (laptop), y en el caso de que el dispositivo funcione en forma autónoma desconectado de esta, se le agrega un reloj a la placa Arduino.

3) Se establece la hora de apertura o cierre de las celosías según la preferencia de los usuarios, dato tomado a través de las encuestas.

4) Se activa el paquete de códigos que mandan la orden de apertura de la celosía, parámetro True False en programación algorítmica.

5) Se activa el paquete de códigos que mandan la orden de cierre la de celosía, parámetro True False en programación algorítmica.

6) Se activa la animación en Rhino de lo que está sucediendo con las celosías, y es necesario que esté conectada la laptop. Esto punto es solo para monitoreo y futuras correcciones con los usuarios.

Si bien en la prueba de campo se usó una laptop conectada al Arduino para hacer las correcciones, el objetivo final es dejar el dispositivo trabajando en forma autónoma sin estar conectado a esta. Esto se puede hacer ya que placa Arduino tiene una memoria y se puede dejar grabada las ordenes de la programación y por otro lado se le tendría que agregar un reloj y una batería externa, como la que se usan para la carga de celulares. Esta batería se conecta al dispositivo a través de un cable USB. La importancia de agregar un reloj es fundamental, ya que este es el que define el momento en que los dedos robóticos deben accionar sobre el control remoto.

Con respecto al análisis post-ocupacional se basaron tanto en aspectos cuantitativos como cualitativos. En los primeros se tomaron mediciones con sensores luminotécnicos Tipo Hobbo durante una semana, en la oficina que presentaba mayores quejas de deslumbramiento por parte de los usuarios. Estos sensores miden y registran la cantidad de iluminación natural recibida cada cierta frecuencia, que en este caso era cada 15 minutos.

Toda esta cantidad de datos expresados en Lux, se exportan a una planilla Excel para luego ser leída desde la programación del 


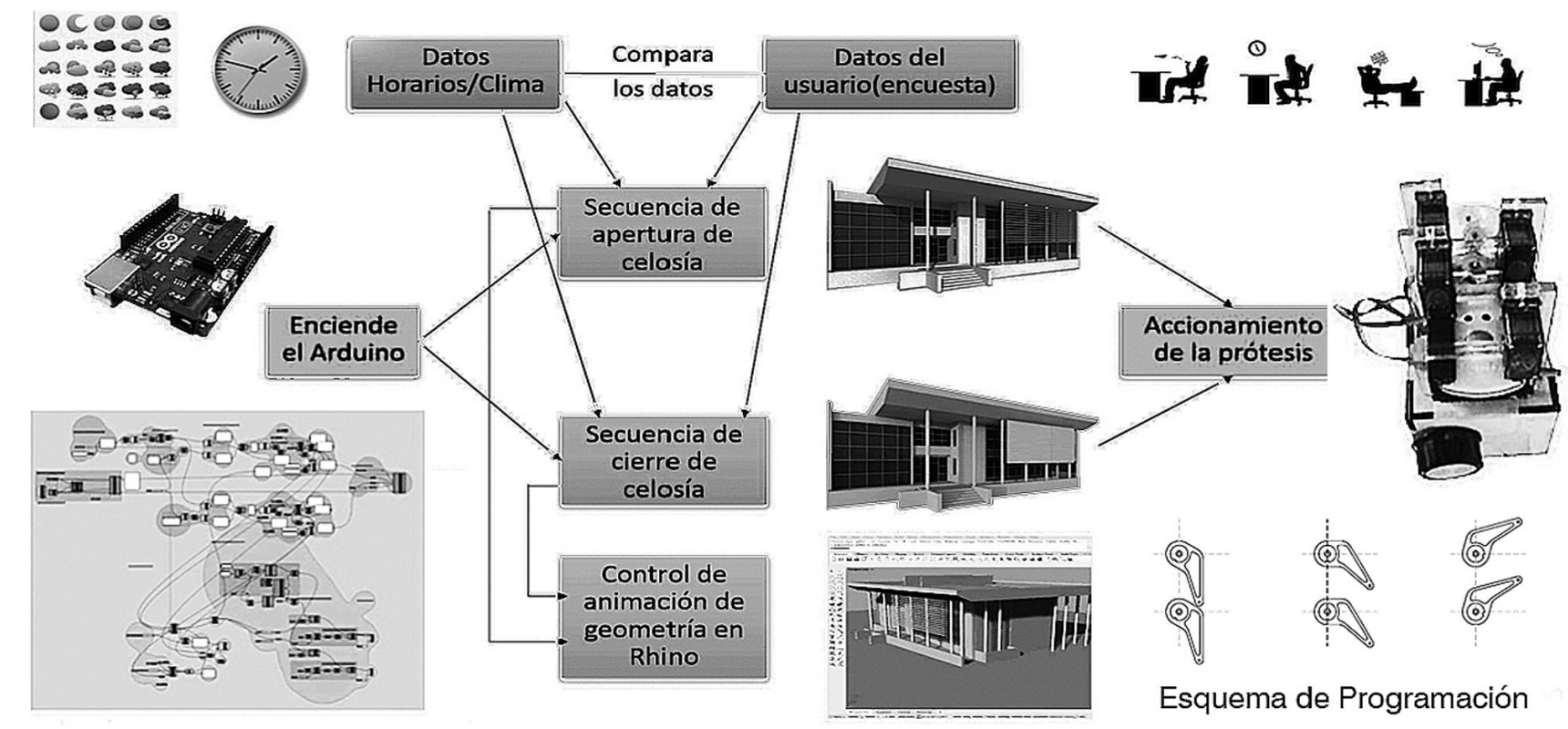

Figura 3: Esquema Programación (arduino, grasshopper, firefly)

grasshopper. Una de las finalidades que se pensó primeramente era operar los dedos robóticos según un óptimo de iluminación natural (dato cuantitativo).

En el análisis cualitativo, se optó por realizar entrevistas a los mandantes y encuestas a los usuarios. Con los primeros, la idea era poder tener un panorama de cuáles eran las premisas de cómo debía funcionar el edificio cuando lo proyectaron, y contraponer esto con las encuestas a los usuarios para ver cómo ellos percibían su funcionamiento.

Dicha encuesta se centró en los factores lumínicos principalmente, excluyendo los factores térmicos o acústicos ya que el uso de las celosías en este caso en particular del CITEC no gravita sobre estos. Por otro lado se les consulto a los usuarios sobre la percepción que tenían de la imagen del edificio como representación de las actividades que allí se realizan (Centro de Investigación Tecnológica). Esto es debido a que este desarrollo pertenece a una tesis de maestría que se debate entre la tensión de lo simbólico-expresivo de la arquitectura vs el desempeño funcional.

El resultado de la encuesta fue que hay un problema de deslumbramiento principalmente en una oficina del primer piso y más precisamente sobre una persona que es la que se ve más afectada. Se comprobó que dicha persona es al final la que termina operando el control remoto para abrir o cerrar la celosía según el grado de disconfort que el deslumbramiento le genere. Procesando todos estos datos, se llegó a la conclusión que el mayor grado de deslumbramiento se produce en invierno, principalmente de 9 a 10 hs a.m. debido al azimut y altitud del Sol (Concepción, Chile) y como ingresan los rayos solares a la oficina. Este dato fue clave para la programación de los dedos robóticos, lo cuales a esa hora tienen que operar sobre el control remoto para cerrar las celosías. Se procedió a la manufactura de la prótesis robótica, ajustes e implementación del dispositivo propuesto.

\section{PROGRAMACIÓN VISUAL Y CÓDIGO ABIERTO}

La Programación Visual ha posibilitado a Diseñadores y Arquitectos intervenir sobre sus propios instrumentos proyectuales innovando en los procesos de diseño. La personalización y creación de formas de trabajo mediante scripts permite la generación de instrumentos acordes a las nuevas lógicas propuestas. Es así como la aplicación de secuencias de comandos para la productividad, la experimentación y la especulación en el diseño permite la creación de prototipos dinámicos basados en geometría generativa complementándose con programación de código abierto para la generación de dispositivos diversos. El Diseño Paramétrico introduce la geometría desde una visión matemática-algorítmica. Propone la generación de geometría a partir de la definición de una familia de parámetros iniciales y la programación de relaciones formales entre ellos. En estos procesos, la utilización de algoritmos y recursos computacionales avanzados no se utilizan solo para representar formas y controlar geometrías complejas creando posibilidades proyectuales dinámicas y variables, sino que además posibilita intervenir en la construcción de dispositivos mecánicos simples mediante microcontroladores con entornos de desarrollo de código abierto.

\section{ARQUITECTURA Y ENSEÑANZA: REDES LOCALES Y REGIONALES}

La arquitectura, como profesión en el mundo contemporáneo, se caracteriza por ser múltiple, diversa, compleja y contradictoria, cercada por variados intereses y presiones de orden económico, político y social. La presencia social contemporánea del arquitecto se 


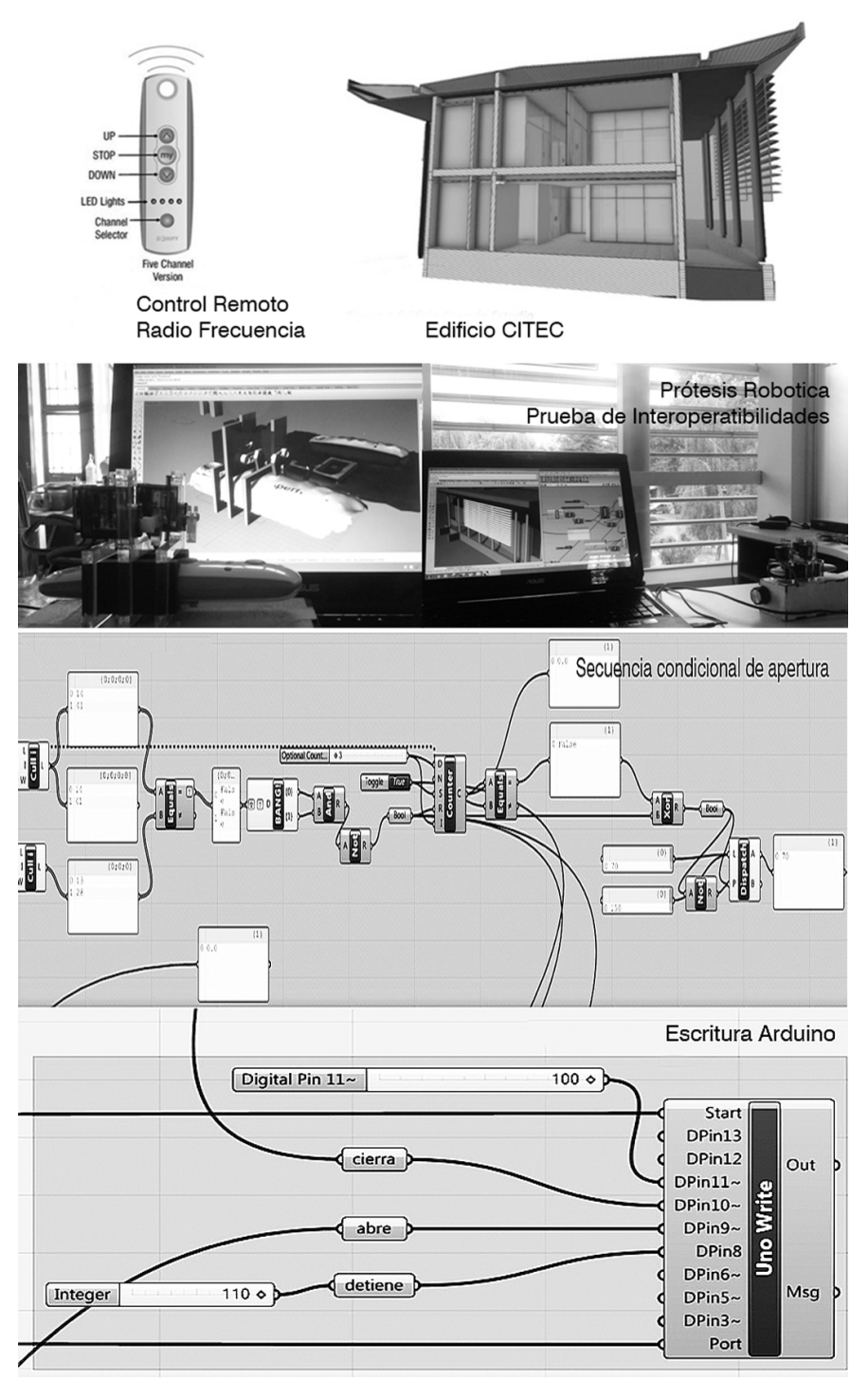

Figura 4: Pruebas in situ de Interoperatibilidades + programación visual

manifiesta también en múltiples imágenes: el empresario exitoso; el jefe de un sector específico de una gran empresa; el director de un departamento público; el profesional independiente con clientes individuales; el asesor y crítico de arte; coordinador de equipos interdisciplinarios; empleado; asesor; emprendedor; artista del ciberespacio. Cada uno representa diferentes grados de valoración, incumbencias y reconocimientos en lo profesional, en lo económico y lo cultural. Se desprende de ello lo dificultoso de organizar y evaluar con iguales principios y parámetros, las diferentes producciones realizadas por los singulares perfiles y circunstancias dadas. La imposibilidad de imponer un sistema de validación general que sirva para las diversas situaciones ha marcado a la enseñanza del proyecto contemporáneo en las facultades y escuelas de arquitectura y se potencia hoy en día con las actuales condiciones culturales e instrumentales del sujeto contemporáneo donde la creatividad se amplía en un proceso de intercambio mediante autorías colectivas, bases de datos digitales y construcciones hipertextuales. El arquitecto, inmerso en esta escena contemporánea y conjuntamente valorado socialmente como hacedor de edificios, desafía al panorama de la enseñanza la que es orientada frecuentemente hacia un desarrollo profesional localizado.

\section{CULTURA MAKER ${ }^{7}$ E INTEGRACIÓN GLOBAL}

El movimiento Maker es la habilidad de ser pequeño y al mismo tiempo mundial; artesanal e innovador; de alta tecnología y de bajo coste. El movimiento Maker está haciendo por los productos físicos lo que el código abierto hizo por el software. La cultura Maker es una extensión del DIY (do it yourself) basada en la tecnología. Los intereses comunes incluyen desarrollos en electrónica, robótica, impresión 3D y el empleo de máquinas $\mathrm{CNC}$, como así también el uso de herramientas clásicas como el trabajo de herrería, tornería, carpintería y las tradicionales artes y oficios.

Participar de experiencias vinculadas a la Cultura Maker permite una importante salida para la exploración personal, aumenta la comprensión de cómo los medios electrónicos funcionan y compromete a los individuos a ser actores en una cultura que es cada vez más compleja, tecnológica y digitalizada. Permite la construcción de espacios de poder íntimos y personales interactuando con multitudes. Es el poder de una sola persona para dirigir su propia educación, encontrar su propia inspiración, moldear su propio entorno y compartir su aventura con quien esté interesado. Según Chris Anderson ${ }^{8}$ durante la última década se trató de buscar formas de crear, inventar y trabajar juntos en la web. En la próxima década estos aprendizajes se concentrarán en la construcción y manufactura colectiva de objetos del mundo físico. El movimiento Maker está haciendo por los productos físicos lo que el código abierto hizo por el software.

Durante décadas, debido a los conocimientos, el equipamiento y los costos implicados en producir objetos a gran escala, la fabricación ha sido sobre todo una cuestión de grandes empresas y profesionales muy bien formados. Hoy en día las empresas nacen en dormitorios de estudiantes y se hacen grandes antes de que sus fundadores se gradúen. Basándose en la fabricación digital de alta tecnología, es posible que individuos emprendedores puedan utilizar a las grandes empresas a voluntad para realizar lo que deseen. En la variabilidad está el desafío y la impronta del Diseño personalizado.

La cultura Maker enfatiza el aprendizaje colaborativo y la cognición distribuida. La interacción en la comunidad y el intercambio de conocimientos se haya mediado por las tecnologías de trabajo en red. Los sitios web y las redes sociales son la base del repositorio de conocimientos y el canal central del intercambio de ideas e información, hibridado por encuentros físicos en espacios de intercambio social como los Hackerspaces y los Fab Lab. Impulsados principalmente por la llegada de la impresión 3D para la fabricación de prototipos, la disminución de costos y la gran aceptación de éstas tecnologías, se fueron consolidando nuevos campos de innovación. La nube en sí es una herramienta al servicio del movimiento creador, lo que permite una mayor colaboración, flujo de trabajo digital, y la manufactura compartida mediante la descarga y distribución de archivos. Mientras tanto, el movimiento de código abierto, que se había 

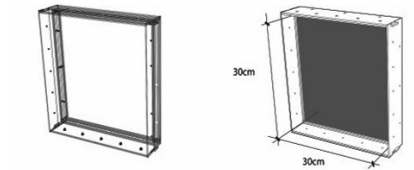

Area de trabajo $30 \times 30 \mathrm{~cm}$
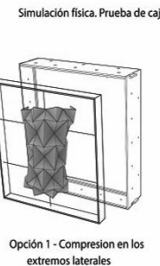

extremos laterales
ysuperior enterior

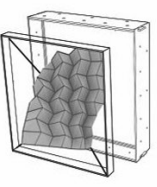

Opcion 2 - Tension en los
Euatros extremos, alternando
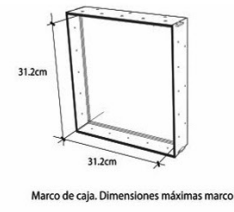
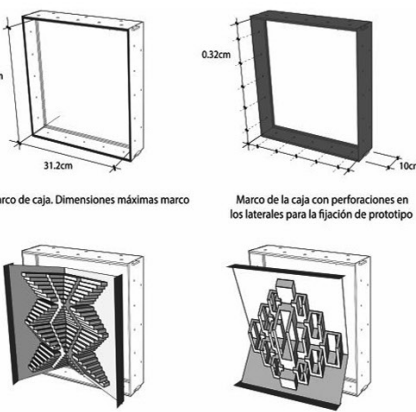

Opcibn 3-Rigidezen los
laterales zizquierdoyderecho
Rettrimiento de los lodos

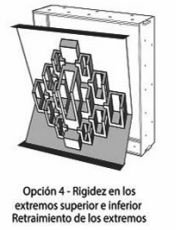

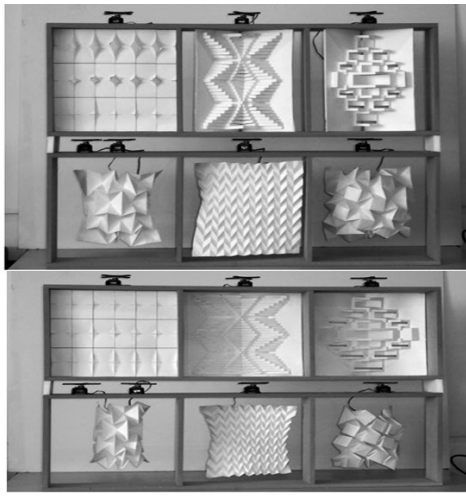

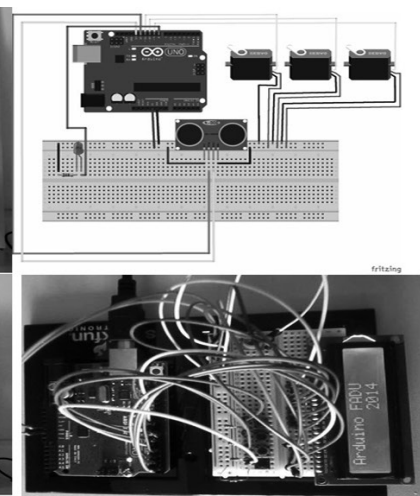

Figure 5: Programación Visual General (grasshopper, firefly)

centrado inicialmente en el software, se ha ido expandiendo en el hardware, asistido por el fácil acceso a los planos en línea y acuerdos de licencia.

\section{EXPERIENCIAS COLABORATIVAS}

La sociedad de la información y las nuevas tecnologías potencian metodologías participativas y aprendizajes colaborativos. El aprendizaje colaborativo y el aprendizaje situado, coinciden en que todo aprendizaje tiene lugar en un contexto en el que los participantes negocian los significados. Así el escenario de aprendizaje se constituye en un campo de interacción de ideas, representaciones y valores en donde los sujetos, individualmente, obtienen diferentes interpretaciones de las mismas experiencias construyendo y reconstruyendo sus conocimientos según saberes, esquemas, y vivencias personales ${ }^{9}$.

El principal cambio de paradigma que proponen las redes globales en la sociedad de la información es el abandono de la figura tradicional de un único transmisor de conocimiento para abordar la autogestión del mismo, su correlación y puesta en práctica por sujetos concentrados en el diseño de sus propios procesos. De esta forma ya no es prioridad establecer solo una base de conocimiento en el individuo, en una memoria privada que uno debe llenarla con cosas que va usar en el futuro. Desde un concepto de memoria pública (bases de datos, redes interconectadas) la información ya se encuentra disponible y asequible en tiempo real, entonces su prioridad ahora es como llegar a ella, como usarla, como reciclarla. Se trata de privilegiar el análisis y la interpretación de la información sobre la acumulación de datos. Una apuesta por el conocimiento y la creatividad frente a las tradicionales técnicas de reproducción mnemotécnica de procedimientos o conceptos. Es así como nuestra disciplina incorpora nuevos instrumentos para una actuación mas estratégica como mediador dentro de una amplia red de posibilidades y nuevas incumbencias disciplinares repensando procesos conocidos e incorporando valiosos saberes transdisciplinares. Este trabajo ha expuesto una particular experiencia de adaptación del concepto de piel dinámica a la realidad latinoamericana mediante prótesis robóticas que evidencian lógicas de cultura maker de códigos abiertos para contextualizar sistemas industriales cerrados, encarando la localización de lo global.

\section{AGRADECIMIENTOS}

Personal Técnico y Administrativo CITEC-UBio-Bio; DAU: Doctorado en Arquitectura y Urbanismo, UBio-Bio. Proyecto PICT2014-3567 (FonCyT, ANPCYT).

\section{NOTAS}

1. Gausa Manuel y Otros (2001). Diccionario Metápolis de Arquitectura Avanzada. Ed. Actar. Barcelona

2. Chiarella, M; Raffin, A; Martini, S; Gongora, N; Bressan, F. Pieles Arquitectónicas Dinámicas. Prototipos a escala mediante prototipado rápido, microcontroladores y patrones plegados., p. 96-100. In: Proceedings of the XVIII Conference of the Iberoamerican Society of Digital Graphics: Design in Freedom. Montevideo. 2014

3. Ataman O; Rogers J. and Ilesanmi A. Redefining the Wall: Architecture, Materials and Macroelectronics. International Journal of Architectural Computing 4, no. 4. pag. 125-136. 2006

4. Glynn, R. Conversational environments Revisited, Bartlett School of Architecture, UCL, Wates house, Londres. Cybernetic conference, 2008.

5. Fox M.; Kemp M. (2009). Interactive Architecture. Princeton Architectural Press.

6. Meir I; Garb Y; Jiao D; Cicelsky; A. Post-Occupancy Evaluation: An Inevitable Step Toward Sustainability. Advances in Building Energy Research. Vol. 3, Iss. 1, 2009

7. Anderson, C. (2013). Makers: The New Industrial Revolution. Crown Business.

8. Ibid

9. Chiarella, M; Garcia Alvarado, R.; Martín-Pastor, A. (2016). Proyectación y Construcción Digital. En L. Muller, Reffinetti Martins, M.L. (Eds), Arquitetura e qualidade socioambiental nas cidades do Cone Sul. (Cap III, pp. 431-445). Programa CAPF-BA. (CAPES-SPU). USP-UNL. Ediciones UNL. Argentina 